Research Article

\title{
Evaluating the Dynamic Response of the Bridge-Vehicle System considering Random Road Roughness Based on the Moment Method
}

\author{
Fan Feng $\mathbb{D}^{1},{ }^{1}$ Fanglin Huang $\mathbb{D}^{1},{ }^{1}$ Weibin Wen $\mathbb{D}^{1},{ }^{1}$ Zhe Liu $\mathbb{D}^{2},{ }^{2}$ and Xiang Liu $\mathbb{D}^{3}$ \\ ${ }^{1}$ School of Civil Engineering, Central South University, Changsha 410075, China \\ ${ }^{2}$ School of Business Administration, Henan University of Animal Husbandry and Economy, Zhengzhou 450044, China \\ ${ }^{3}$ School of Civil Engineering, Fujian University of Technology, Fuzhou 350118, China
}

Correspondence should be addressed to Xiang Liu; liuxiang@fjut.edu.cn

Received 10 March 2021; Accepted 15 October 2021; Published 1 November 2021

Academic Editor: Valeria Vignali

Copyright (c) 2021 Fan Feng et al. This is an open access article distributed under the Creative Commons Attribution License, which permits unrestricted use, distribution, and reproduction in any medium, provided the original work is properly cited.

The bridge-vehicle interaction (BVI) system vibration is caused by the vehicles passing through the bridge. The road roughness has a great impact on the system vibration. In this regard, poor road roughness is known to affect the comfort of the vehicle crossing the bridge and aggravate the fatigue damage of the bridge. Road roughness is usually regarded as a random process in numerical calculation. To fully consider the influence of road roughness randomness on the response of the BVI system, a random BVI model was established. Thereafter, the random process of road roughness was expressed by Karhunen-Loeve expansion (KLE), after which the moment method was used to calculate the maximum probability value of the BVI system response. The proposed method has higher accuracy and efficiency than the Monte Carlo simulation (MCS) calculation method. Subsequently, the influences of vehicle speed, roughness grade, and bridge span on the impact factor (IMF) were analyzed. The results show that the road roughness grade has a greater impact on the bridge IMF than the bridge span and vehicle speed.

\section{Introduction}

The bridge-vehicle interaction (BVI) vibration caused by vehicles passing through the bridge will have a direct impact on the working state and service life of the bridge. It is an important design measure to evaluate the structural design parameters such as the stability and safety of vehicles running on the bridge $[1,2]$. The main causes of the BVI vibration are vehicle dynamic characteristics, road roughness, vehicle braking, vehicle speed, dynamic characteristics of the bridge's structure, bridgehead overlap, bridge deck local potholes, vehicle driving position, etc.

Road roughness is an important factor affecting the BVI vibration. The influence of road roughness, which has strong randomness, cannot be ignored when calculating the BVI vibration. Road roughness is the main excitation source of the BVI vibration. Generally, road roughness is considered a stationary Gaussian random process with zero-mean value wherein roughness can be characterized by power spectrum density (PSD). Oliva et al. [3] proposed an algorithm for generating road roughness samples with correlation based on Fourier transform. On the basis of this method, Zou et al. [4] studied the influence of the sample correlation coefficient of roughness on the vibration response of the BVI system, pointing out that there are obvious differences in the calculated responses of the BVI system under different road roughness.

At present, there are many achievements in the study of BVI vibration. Zou et al. [5] proposed a method for predicting the bounds of the BVI responses with uncertain bridge and vehicle parameters, whereas Wang et al. [6] estimated the road roughness based on the BVI system. Similarly, Zhong et al. [7] analyzed the BVI dynamic response considering the foundation settlement, while Liu et al. [8] analyzed the safety of vehicles running on the bridge during earthquakes. Xu et al. $[9,10]$ analyzed the vehicle- 
track coupled system based on the multi-finite-element method. Xin et al. [11] analyzed the resonance of bridge considering the randomness of parameters, and Xin et al. [12] examined the uncertainty and sensitivity of parameters of the vehicle-bridge system. Meanwhile, Xiang et al. [13] analyzed the vehicle-bridge coupled dynamic system after considering the creep effect.

Generally, the response of the BVI system in consideration of road roughness can be calculated from the aspect of the random dynamic theory. In this context, $\mathrm{Wu}$ and Law [14] proposed a new method for dynamic analysis of the BVI considering uncertainty, in which the Karhunen-Loeve expansion (KLE) was applied to deal with the random field of road roughness. In addition, the statistical moment of system response was solved by the spectral method; the results showed that the method has high accuracy and efficiency. Then, $\mathrm{Wu}$ and Law [15] used a similar method to calculate the dynamic response of the BVI that considered the stochastic field of bridge structure parameters [15], vehicle axle load identification [16], and random system response calculation [17]. On the other hand, Chen et al. $[18,19]$ and Li et al. [20] proposed an efficient method to calculate the static and random response of the structural system. Xu et al. [21-25] carried out extensive research on the vehicle-bridge coupling vibration considering track irregularity. In the studies conducted by Liu et al. [26-28], KLE was also used to represent the stochastic process and combined with the point estimate method (PEM) $[29,30]$. Thereafter, a new stochastic FEM approach and the dynamics response of vehicle and bridge were analyzed.

In order to study the dynamic response of vehicles crossing the bridge that fully considers the randomness of the road roughness, the moment method is applied to calculate the BVI system, in which KLE is used to mathematically express the random process of road roughness, whereas the PEM is used to calculate the statistical moment of the response. The system response can be evaluated quickly and easily by combining KLE and PEM methods (called KLE-PEM).

\section{Dynamic Equation of the Bridge- Vehicle System}

When a vehicle crosses a bridge, the two interact. Therefore, in the dynamic response analysis of the vehicle passing the bridge, the two are usually regarded as a system for analysis, which is referred to as the BVI system [31].

As shown in Figure 1, a car moves at a speed on a simply supported beam. The model of the vehicle is regarded as a mass-spring-dashpot system, which includes one car body, two wheel-axles, and two wheels. The car body has two degrees of freedom (DOFs), namely, vertical and nod displacement. Each wheel-axle has only one DOF. The wheel is supposedly in close contact with the road, which means that the wheel has no independent DOFs. Therefore, the whole vehicle system has four DOFs.

The FEM is used to model the bridge, in which the bridge damping matrix adopts Rayleigh damping. According to the energy law and considering the damping force of the system, the total potential energy of the BVI system can be obtained, which is expressed as follows:

$$
\Pi_{d}=U_{i}+V_{m}+V_{F}+V_{p}+V_{g}+V_{c},
$$

where $U_{\mathrm{i}}$ is the strain energy of the BVI system; $V_{\mathrm{m}}$ refers to the negative work done by the inertia force of the system and $V_{m}=-\int_{v}\left(-u^{T} \rho \ddot{u}\right) \mathrm{d} v ; V_{\mathrm{c}}$ denotes the negative work done by damping force and $V_{c}=-\int_{v}\left(-u^{T} c \ddot{u}\right) \mathrm{d} v ; V_{\mathrm{F}}$ is the negative work done by Coulomb friction of the system and $V_{F}=-u F$ sign $\dot{u} ; V_{\mathrm{p}}$ signifies the negative work done by external force of the system and $V_{p}=-u^{T} P$; and $V_{\mathrm{g}}$ is the system gravitational potential energy and $V_{g}=-u^{T} Q$.

The dynamic equation of the system can be obtained quickly by combining the first-order variation of the total potential energy $\Pi_{d}$ and the principle of elastic potential energy invariance $\delta \Pi_{d}=0$ [32]. The dynamic equation of the BVI can be written as follows:

$$
\left[\begin{array}{cc}
M_{v v} & 0 \\
0 & M_{b b}
\end{array}\right]\left\{\begin{array}{l}
\ddot{X}_{v} \\
\ddot{X}_{b}
\end{array}\right\}+\left[\begin{array}{cc}
C_{v v} & C_{v b} \\
C_{b v} & C_{v b}
\end{array}\right]\left\{\begin{array}{c}
\dot{X}_{v} \\
\dot{X}_{b}
\end{array}\right\}+\left[\begin{array}{cc}
K_{v v} & K_{v b} \\
K_{b v} & K_{b b}
\end{array}\right]\left\{\begin{array}{c}
X_{v} \\
X_{b}
\end{array}\right\}=\left\{\begin{array}{l}
F_{v} \\
F_{b}
\end{array}\right\},
$$

where $M, C$, and $K$ represent mass matrix, damping matrix, and stiffness matrix, respectively; $\ddot{X}, \dot{X}$, and $X$ denote acceleration vector, velocity vector, and displacement vector, respectively; the subscript $v v$ or $v$ denotes vehicle; $b b$ or $b$ signifies bridge; and $v b$ or $b v$ is the coupling part of bridge and vehicle. $F$ denotes the force vector, whereas $F_{\mathrm{v}}$ signifies the force vector of the vehicle caused by the displacement and the first derivative of road roughness, which can be written as

$$
F_{v}=\left[\begin{array}{llll}
0 & 0 & F_{t 1} & F_{t 2}
\end{array}\right]^{T},
$$

with $F_{t 1}=k_{t 1} r_{t 1}(t)+c_{t 1} \dot{r}_{t 1}(t)$ and $F_{t 2}=k_{t 2} r_{t 2}(t)+c_{t 2} \dot{r}_{t 2}$ $(t)$, where $r_{t 1}(t)$ and $r_{t 2}(t)$ denote the roughness of front and rear axle positions at time $t$, and $\dot{r}_{t 1}(t)$ and $\dot{r}_{t 2}(t)$ denote the first derivative of the roughness of front and rear axle positions at time $t . F_{\mathrm{B}}$ denotes the force vector of the bridge caused by road roughness and axle load of the vehicle, which can be expressed as

$$
F_{B}=F_{t}+F_{g}
$$

with 


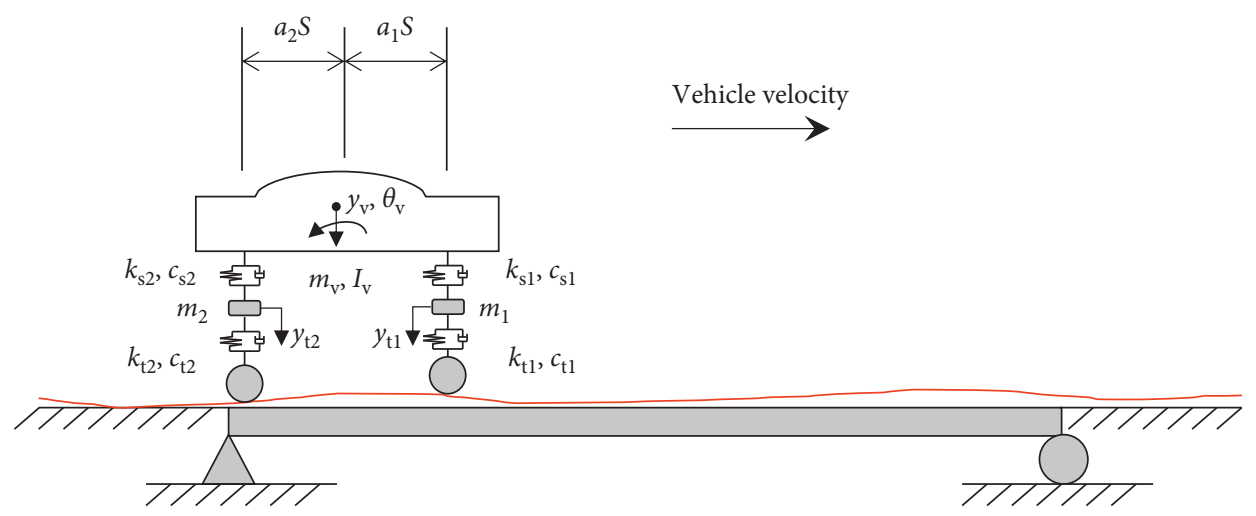

Figure 1: The BVI system.

$$
\begin{aligned}
& F_{B k}=-\left(F_{t 1} N_{t 1}+F_{t 2} N_{t 2}\right) \text {, } \\
& F_{g}=-\left[\left(m_{1}+a_{1} m_{v}\right) g N_{t 1}+\left(m_{1}+a_{2} m_{v}\right) g N_{t 2}\right], \\
& N_{t 1}=\left[\begin{array}{llllllllll}
0 & \cdots & 0 & N^{1}{ }_{t 1} & N^{2}{ }_{t 1} & N^{3}{ }_{t 1} & N^{4}{ }_{t 1} & 0 & \cdots & 0
\end{array}\right]^{T} \text {, } \\
& \text { and } N_{t 2}=\left[\begin{array}{llllllllll}
0 & \cdots & 0 & N^{1}{ }_{t 2} & N^{2}{ }_{t 2} & N^{3}{ }_{t 2} & N^{4}{ }_{t 2} & 0 & \cdots & 0
\end{array}\right]^{T} \text {, }
\end{aligned}
$$

where $N_{t i}^{m}$ denotes the shape function of the bridge at the position of front or rear axle, which can be written as

$$
\left\{\begin{array}{l}
N_{t 1}^{m}=1-3\left(\frac{\xi}{l e}\right)^{2}+2\left(\frac{\xi}{l e}\right)^{3}, \\
N_{t 2}^{m}=\xi\left[1-2\left(\frac{\xi}{l e}\right)+\left(\frac{\xi}{l e}\right)^{2}\right], \\
N_{t 3}^{m}=3\left(\frac{\xi}{l e}\right)^{2}-2\left(\frac{\xi}{l e}\right)^{3}, \\
N_{t 4}^{m}=\xi\left[\left(\frac{\xi}{l e}\right)^{2}-\left(\frac{\xi}{l e}\right)\right],
\end{array}\right.
$$

where $\xi$ is the distance between the axle and the left node of the element and le signifies the length of the element.

Equation (2) will be changed by the movement of the vehicle. Therefore, the system is a time-varying system, whereas the Newmark- $\beta$ integral method can be used to calculate the BVI system response.

\section{Dynamic Statistics Calculation Approach}

The Karhunen-Loeve expansion (KLE) is applied as an expression method to simulate the random process of road roughness. The basic expression can be written as follows:

$$
\begin{aligned}
r(x, \theta) & =\bar{r}(x, \theta)+\widetilde{r}(x, \theta) \\
& =\bar{r}(x, \theta)+\sum_{i=1}^{M} \sqrt{\lambda_{i}} \xi_{i}(\theta) \varphi_{i}(x),
\end{aligned}
$$

where $\bar{r}(x, \theta)$ denotes the mean value of road roughness process; $\lambda_{i}$ and $\varphi_{i}(x)$ refer to the $i^{\text {th }}$ eigenvalue and eigenfunction of the covariance kernel, respectively; and $\xi_{i}(\theta)$ denotes the $i^{\text {th }}$ random variable, which is a set of uncorrelated random variables and can be expressed as follows:

$$
\xi_{i}(\theta)=\frac{1}{\sqrt{\lambda_{i}}} \int_{D} \tilde{r}(x, \theta) \varphi_{i}(x) \mathrm{d} x .
$$

Because the road roughness is assumed to be a zeromean Gaussian random process in this paper, $\xi_{i}(\theta)$ will be a set of uncorrelated random normal variables. Equation (7) can be transformed as

$$
r(x, \theta)=\sum_{i=1}^{M} \sqrt{\lambda_{i}} \xi_{i}(\theta) \varphi_{i}(x) .
$$

After obtaining enough samples of road roughness, we can use the method introduced in [26] to solve $\lambda_{i}$ and $\varphi_{i}(x)$ numerically.

According to the theory of statistical moment calculation, the first two central moments of the random system can be calculated by the following formula:

$$
\left\{\begin{array}{l}
\text { MEAN }=\int_{-\infty}^{\infty} g(X) p(x) \mathrm{d} x, \\
\operatorname{VAR}=\int_{-\infty}^{\infty}[g(x)-\operatorname{MEAN}]^{2} p(x) \mathrm{d} x .
\end{array}\right.
$$

where MEAN and VAR denote mean value and variance value, respectively.

Because the integral in (10) is continuous, the result of MEAN and VAR can be obtained by the Gauss integral. By utilizing the dimension reduction technique, the problem of calculating the statistical moment of a system with multiple random variables can be transformed into the problem of calculating the statistical moment of a composite system with a single random variable [27]. Thereafter, the calculation of (10) can be converted to the calculation of the following formula: 


$$
\begin{aligned}
\text { MEAN }= & \sum_{i=1}^{n} E\left[g_{i}\left(X_{i}\right)\right]-(n-1) g(c), \\
\operatorname{VAR}= & \sum_{i=1}^{n} E\left\{\left[g_{i}\left(X_{i}\right)-\operatorname{MEAN}\right]^{2}\right\} \\
& -(n-1)[g(c)-\mathrm{MEAN}]^{2},
\end{aligned}
$$

with

$$
\begin{aligned}
E\left[g_{i}\left(X_{i}\right)\right]= & \sum_{l=1}^{r} \frac{w_{\mathrm{GH}, l}}{\sqrt{\pi}} g_{i}\left(\sqrt{2} x_{\mathrm{GH}, l}\right), \\
E\left[\left(g\left(X_{i}\right)-\mathrm{MEAN}\right)^{2}\right]= & \sum_{l=1}^{r} \frac{w_{\mathrm{GH}, l}}{\sqrt{\pi}} \\
& \cdot\left[g_{i}\left(\sqrt{2} x_{\mathrm{GH}, l}\right)-\mathrm{MEAN}\right]^{2},
\end{aligned}
$$

where $r$ denotes the number of quadrature points, whereas $x_{\mathrm{GH}, 1}$ and $w_{\mathrm{GH}, 1}$ represent the abscissa and weight of Gaussian-Hermite (G-H) quadrature, respectively. The detailed values of G-H are listed in Table 1. A more detailed derivation process can be found in $[27,34]$.

By combining the KLE and PEM (called KLE-PEM), the response of the BVI system can be easily and quickly obtained.

\section{Simulation of Roughness}

Road roughness spectrum recommended in Chinese code GB7031-2005 [35] was used as the roughness power spectral density (PSD) function to simulate the roughness samples, which can be written as follows:

$$
G_{q}(n)=G_{q}\left(n_{0}\right)\left|\frac{n}{n_{0}}\right|^{-w},
$$

where $n_{0}$ is the spatial reference frequency and the value is $0.1 \mathrm{~m}^{-1} ; G_{q}\left(n_{0}\right)$ denotes the road roughness PSD at the spatial frequency of $n_{0}$, and its value is related to the road roughness class; $w$ is the frequency index, which determines the frequency structure of road roughness spectrum, wherein generally $w=2$; and $n$ represents a spatial frequency in the effective frequency band of spatial frequency and signifies the number of cycles of waves contained in each meter of length, with its bandwidth being $(n 1, n 2)$. Meanwhile, $n 1$ and $n 2$ are the upper and lower limits of the effective frequency band, respectively. The bandwidth should ensure that the vehicle vibration kinetic energy caused by road roughness includes the main natural frequency of vehicle vibration when the vehicle is driving at the average speed. In the code GB7031-2005, according to the road PSD, the road surface is divided into eight classes in accordance with the roughness. In this paper, five roughness grades were taken for calculation, as shown in Table 2 .

Using the trigonometric series method (TSM) [16], the PSD function can be transformed into spatial samples of road roughness. Notably, 20,000 road roughness samples
TABLE 1: G-H integration with $r=3$ [33].

\begin{tabular}{lccc}
\hline Point & 1 & 2 & 3 \\
\hline Abscissa $x_{\mathrm{GH}, 1}$ & -1.22474 & 0 & 1.22474 \\
Weight $w_{\mathrm{GH}, 1}$ & 0.29541 & 1.18164 & 0.29541 \\
\hline
\end{tabular}

corresponding to the PSD of class A, class B, class C, class D,

TABle 2: Road roughness coefficient.

\begin{tabular}{lc}
\hline Road grade & Coefficient $G_{q}\left(n_{0}\right) \cdot 10^{-6} \mathrm{~m}^{2} / \mathrm{m}^{-1}$ \\
\hline A: very good & 1 \\
B: good & 6 \\
C: average & 16 \\
D: poor & 64 \\
E: very poor & 256 \\
\hline
\end{tabular}

and class $\mathrm{E}$ were simulated with the length of $120 \mathrm{~m}$ (which can meet the total length of vehicles before entering, during passing, and after leaving the bridge). The roughness samples of different road roughness classes are shown in Figure 2. It can be seen that the roughness range of class $A$ was about -3 to $3 \mathrm{~mm}$. Similarly, the roughness range of class $B$ was about -7 to $7 \mathrm{~mm}$, class $C$ was about -10 to $10 \mathrm{~mm}$, class $\mathrm{D}$ was about -20 to $20 \mathrm{~mm}$, and class $\mathrm{E}$ was about -50 to $50 \mathrm{~mm}$.

The eigenvalues and eigenfunctions of the road roughness samples under different road classes were calculated to obtain the KLE mathematical expression of the random process of road roughness. The first 160 items of the characteristic value of the roughness under each road class are illustrated in Figure 3. Through calculation, the first 115 items of KLE can meet the accuracy requirement of $98 \%$ under different road roughness conditions. Therefore, in the subsequent calculations, 115 was used as the number of truncation terms for each road roughness class; that is, the BVI system has 115 independent random variables.

\section{Numerical Simulation}

5.1. Verification of the BVI Model. In order to verify the accuracy of the BVI model, the results of the classical numerical case in [36] were used to verify the model. In this case, a moving mass-spring system passing through a simply supported beam was calculated. The parameters of the BVI model were set to be consistent with the parameters of the case, subsequent to which the results of the vertical displacement time history response of the midspan are compared with the results of the case, as shown in Figure 4. It can be seen that the results of the current BVI model were essentially consistent with the results of the classic case, thereby verifying the accuracy of the BVI model.

5.2. Comparison with MCS. To verify the applicability of KLE-PEM in the calculation of BVI model, an example was taken in which the bridge was a one-span simply supported beam. Its parameters are shown in Table 3, and the first four mode shapes are shown in Figure 5. The vehicle was a two- 


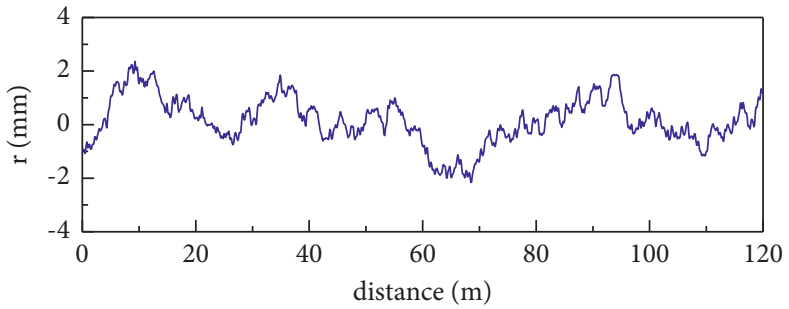

(a)

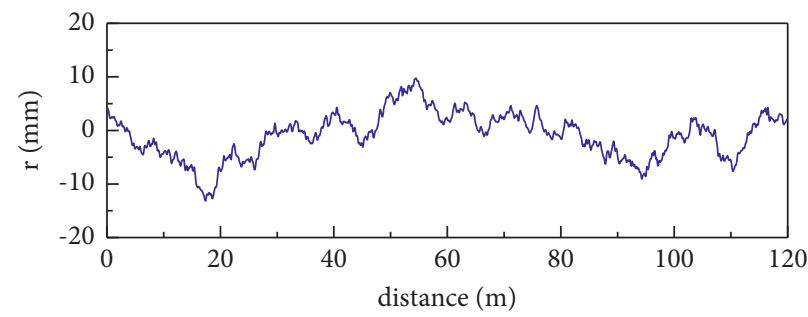

(c)

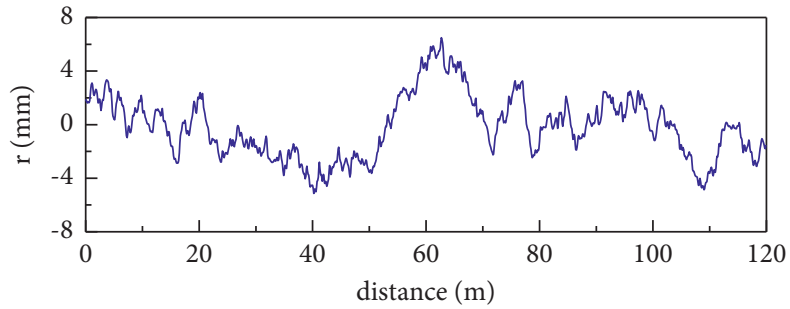

(b)

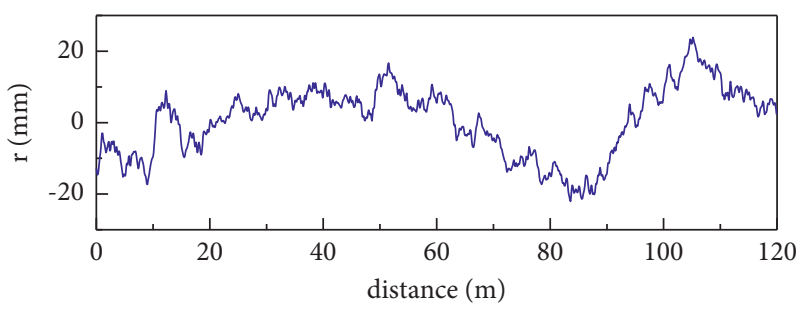

(d)

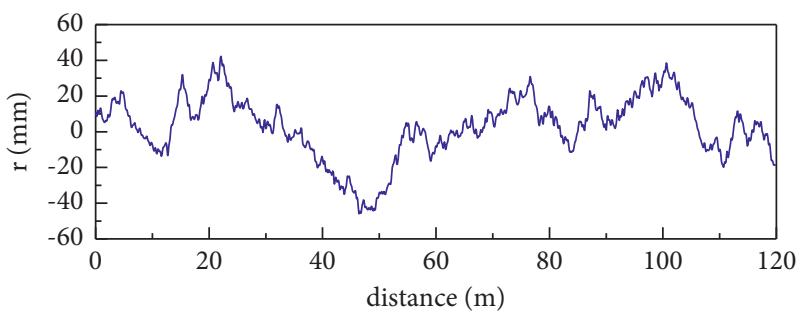

(e)

Figure 2: Road roughness of various roughness classes: (a) class A: very good; (b) class B: good; (c) class C: average; (d) class D: poor; (e) class E: very poor.

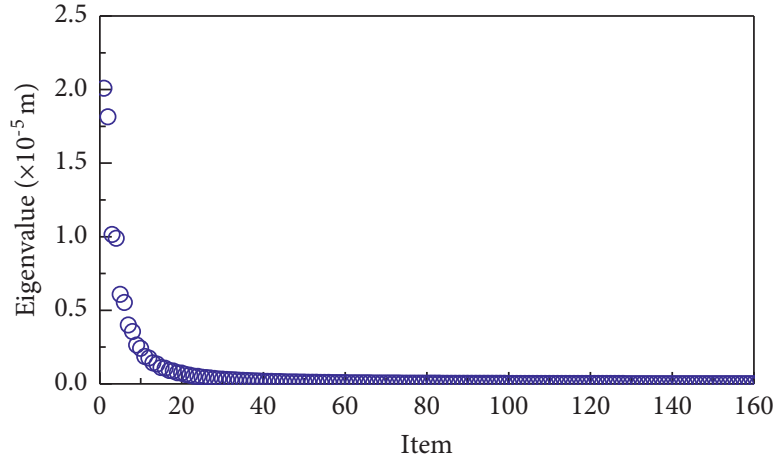

(a)

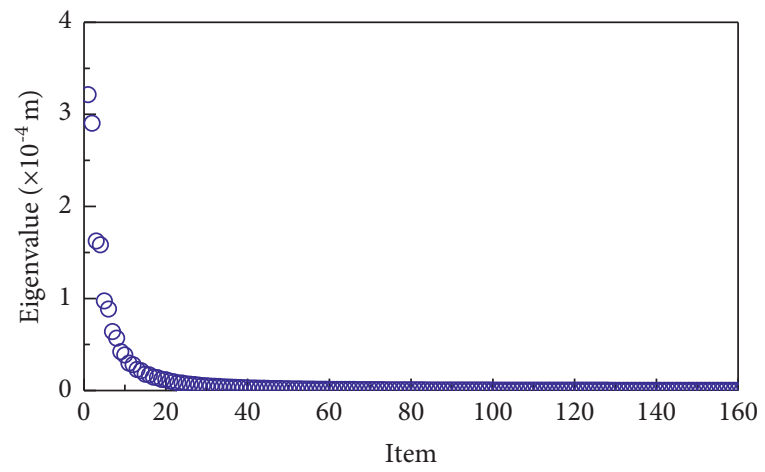

(c)

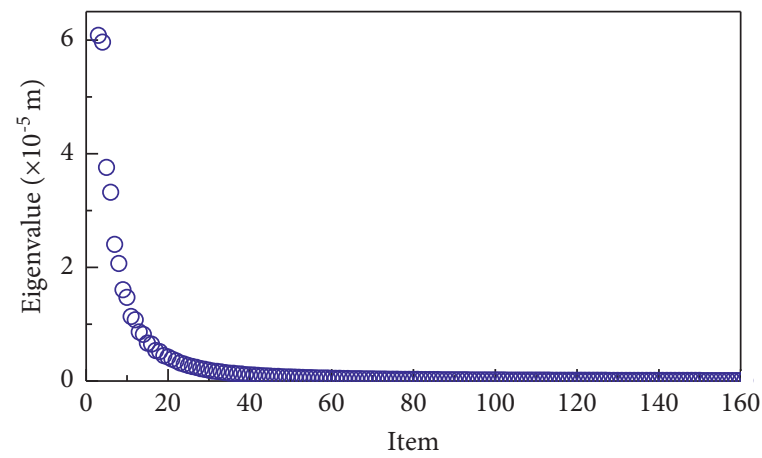

(b)

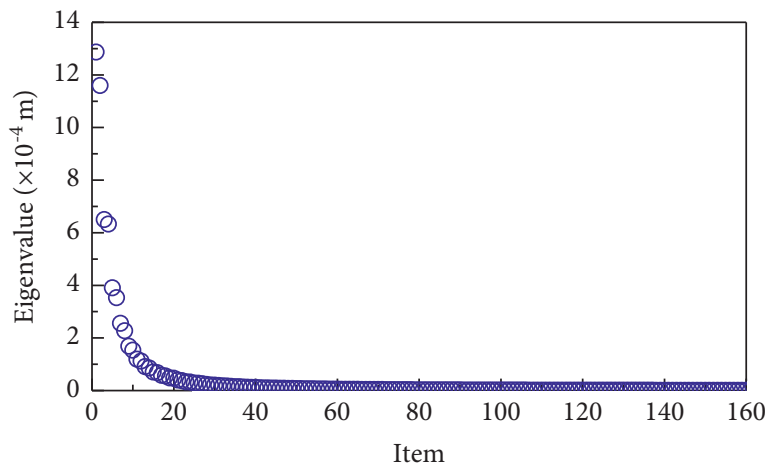

(d)

FIgURE 3: Continued. 


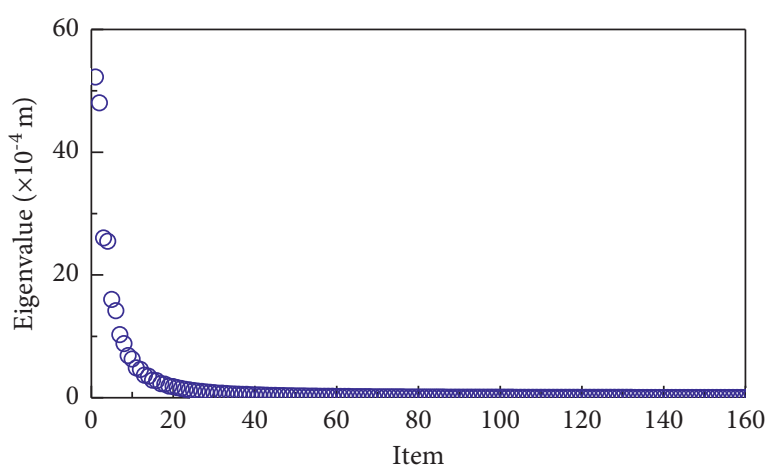

(e)

FiguRE 3: Eigenvalues of each grade of road roughness: (a) class A: very good; (b) class B: good; (c) class C: average; (d) class D: poor; (e) class E: very poor.

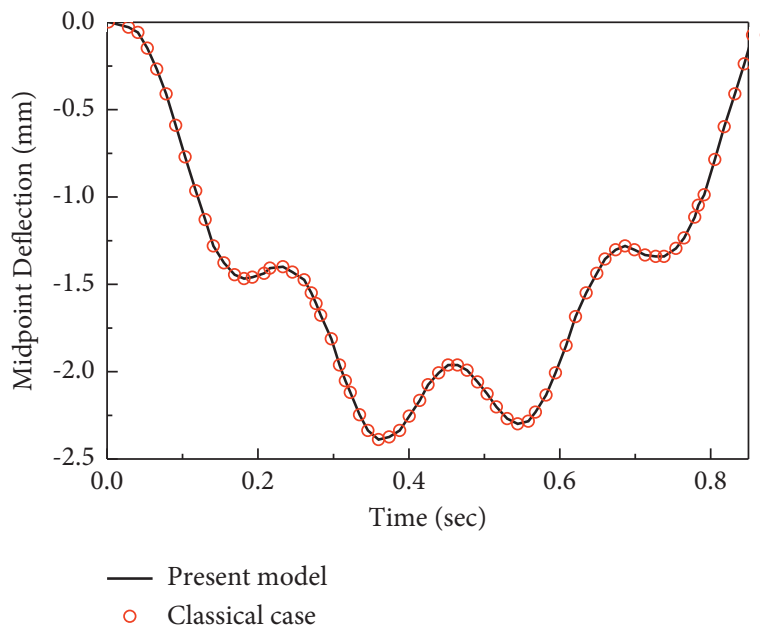

Figure 4: Verification of the BVI model.

TABLE 3: Parameters of the bridge [16].

\begin{tabular}{lcc}
\hline Parameter & Unit & Value \\
\hline Length & $\mathrm{m}$ & 30 \\
Area & $\mathrm{m}^{2}$ & 8 \\
EI & $\mathrm{m}^{4}$ & $2.5 \times 10^{10}$ \\
Density & $\mathrm{kg} / \mathrm{m}^{3}$ & 5000 \\
Poisson's ratio & - & 0.2 \\
Damping ratio & - & $2 \%$ \\
$1^{\text {st }}$ vibration & $\mathrm{Hz}$ & 3.905 \\
$2^{\text {nd }}$ vibration & $\mathrm{Hz}$ & 15.623 \\
$3^{\text {rd }}$ vibration & $\mathrm{Hz}$ & 35.187 \\
$4^{\text {th }}$ vibration & $\mathrm{Hz}$ & 62.721 \\
\hline
\end{tabular}

axle vehicle, and its parameters are displayed in Table 4 . The speed of the vehicle was $20 \mathrm{~m} / \mathrm{s}$.

MCS can be used to calculate the response of a stochastic system as the exact solution to evaluate the accuracy of the new method in a stochastic system. Here, MCS and KLE-PEM were used to calculate the BVI system with class $\mathrm{C}$ road. The number of times BVI programs were called by MCS was 20,000. Since the number of KLE truncation terms of a random process of road irregularity was 115 times, the number of the BVI programs called by KLE-PEM with three estimation points was $115 \times 2+1=231$. The comparison of the mean and variance of the bridge midspan displacement response obtained by calculation is shown in Figure 6. As evidenced by the figure, the results obtained by KLE-PEM coincide with the findings obtained by MCS in terms of mean or variance. In addition, the mean value of response of bridge displacement increased gradually with the passing of vehicles and then decreased gradually after the vehicles left the bridge, finally floating along zero. The variance of bridge displacement response increased when the vehicle moved forward and decreased when the vehicle moved forward. The time when the maximum value appears was essentially consistent with the time when the maximum absolute value of the mean displacement appeared.

The statistical moment results of the vertical acceleration time history response of the car body obtained by the two methods are demonstrated in Figure 7. The statistical moment obtained by KLE-PEM was essentially consistent with that obtained by the MCS method. In addition, for the mean value of response of the vehicle body, the mean value was zero before the vehicle entered the bridge. This is because the road roughness was assumed as a zero-mean random process.

5.3. Bridge Dynamic Response. The dynamic responses of the vehicle passing through the bridge at different vehicle speeds under different road grades were calculated, and the response's maximum probability value was determined by the triple standard deviation criterion [27]. The maximum probability value of vertical displacement response in the middle span of the bridge was obtained, as shown in Figure 8 . It can be seen that the bridge displacement response did not change significantly with the vehicle speed and that the road roughness class had a greater impact on the bridge displacement response. The bridge displacement response increased with the increase of road roughness grade. For class A road, the bridge displacement response was less than $6 \mathrm{~mm}$, while the bridge displacement response was $10 \mathrm{~mm}$ for class D. 


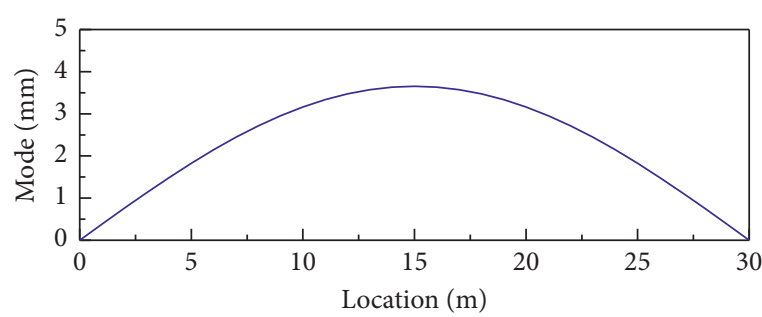

(a)

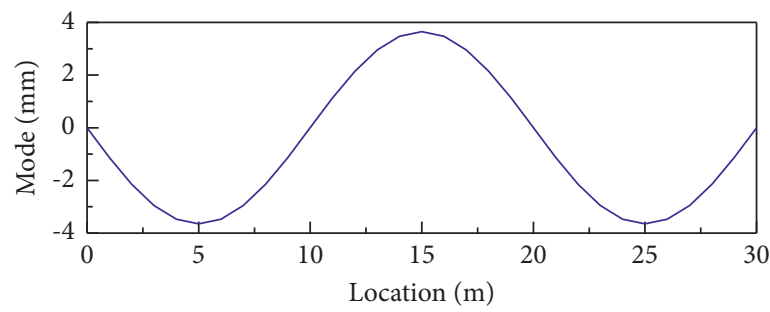

(c)

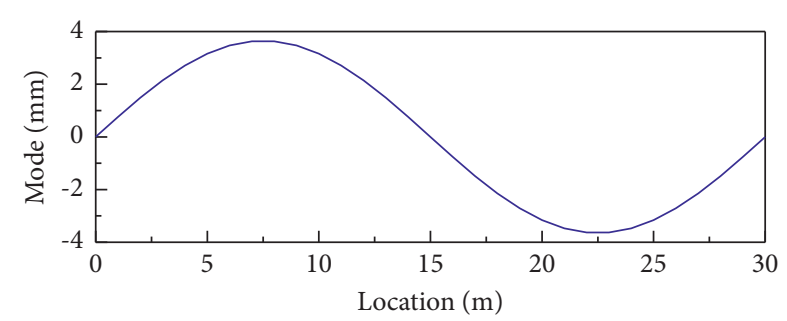

(b)

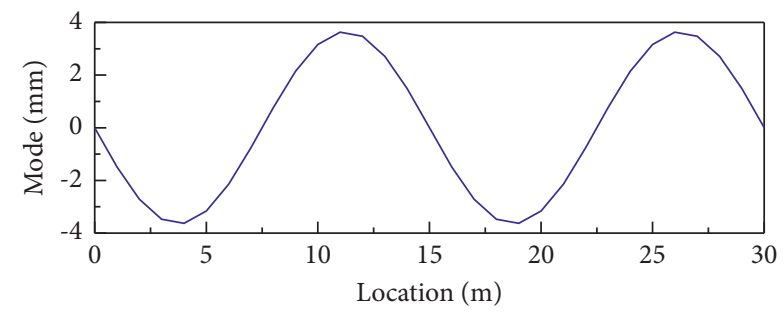

(d)

FIGURE 5: Mode shape of bridge: (a) mode shape 1; (b) mode shape 2; (c) mode shape 3; (d) mode shape 4.

TABle 4: Parameters of the vehicle [16].

\begin{tabular}{lcc}
\hline Symbol & Unit & Value \\
\hline$I_{\mathrm{v}}$ & $\mathrm{kgm}^{2}$ & $1.47 \times 10^{5}$ \\
$m_{\mathrm{v}}$ & $\mathrm{kg}$ & 177,35 \\
$a_{1}$ & - & 0.519 \\
$a_{2}$ & - & 0.481 \\
$m_{1}$ & $\mathrm{~kg}$ & 1500 \\
$m_{2}$ & $\mathrm{~kg}$ & 1000 \\
$k_{\mathrm{s} 1} / k_{\mathrm{s} 2}$ & $\mathrm{~N} / \mathrm{m}$ & $2.47 \times 10^{6} / 4.23 \times 10^{6}$ \\
$k_{\mathrm{t} 1} / k_{\mathrm{t} 2}$ & $\mathrm{~N} / \mathrm{m}$ & $3.74 \times 10^{6} / 4.6 \times 10^{6}$ \\
$c_{\mathrm{s} 1} / c_{\mathrm{s} 2}$ & $\mathrm{~N} / \mathrm{m} / \mathrm{s}$ & $3.0 \times 10^{4} / 4.0 \times 10^{4}$ \\
$c_{\mathrm{t} 1} / c_{\mathrm{t} 2}$ & $\mathrm{~N} / \mathrm{m} / \mathrm{s}$ & $3.9 \times 10^{3} / 4.3 \times 10^{3}$ \\
$\mathrm{~S}$ & $\mathrm{~m}$ & 4.27 \\
\hline
\end{tabular}

The maximum probability value of vertical acceleration response in the middle of the bridge span is shown in Figure 9. Unlike the displacement response, the acceleration response in the middle of the bridge evidently changed with the change of the vehicle speed, whereas the acceleration response first increased and then decreased with the change of the vehicle speed. For bridges with different roughness grades, the maximum acceleration response appeared when the vehicle speed is $20 \mathrm{~m} / \mathrm{s}$. In addition, the acceleration response of the bridge increased with the increase of road roughness grade.

5.4. Vehicle Dynamic Response. The maximum value of the vertical acceleration of the car body calculated by KLE-PEM under different roughness classes without vehicle speed is shown in Figure 10. It can be seen that in the case of different roughness levels, the acceleration of the car changes with the speed. More specifically, it first shows a trend of a decline followed by an increase. When the speed was $20 \mathrm{~m} / \mathrm{s}$, the maximum acceleration of the car body was found to be the smallest. Similarly, the roughness grade of a road surface was found to have a great impact on the acceleration of the car body.
5.5. Impact Factor of the Bridge. Dynamic impact factor (IMF) is generally defined as the ratio of dynamic load effect and static load effect of the bridge under vehicle load; i.e.,

$$
\mu=\frac{\left(S_{d}-S_{s}\right)}{S_{s}},
$$

where $S_{\mathrm{d}}$ is the maximum dynamic response of the bridge under moving vehicle load, $S_{S}$ denotes the maximum static response of the bridge under corresponding vehicle load, and $\mu$ signifies the IMF.

IMF is not only an important index to characterize the impact effect of moving vehicle load on the bridge, but also a parameter affected by multiple factors. In traditional research studies, the dynamic IMF has been calculated by collecting the bridge response from the real bridge test, whereas the empirical calculation formula of IMF was obtained by regression analysis of the collected samples. However, on the one hand, the test method is expensive and difficult to implement; on the other hand, different bridges have different stress conditions, and the measured results of a limited number of bridges may not be widely representative. In contrast, IMF analysis based on numerical simulation has the characteristics of low cost and flexible simulation of different bridges and different working conditions, which is why it is eliciting widespread attention.

In Section 5.3, it can be inferred that road roughness has a great effect on the dynamic response of the bridge, thereby implying that road roughness influences the IMF of the bridge as well. To systematically discuss the IMF of the bridge, the IMFs with different vehicle speeds, different bridge spans, and different road grades were calculated from the random aspect. The range of vehicle speed was from 10 to $35 \mathrm{~m} / \mathrm{s}$; the bridge spans were $20 \mathrm{~m}, 22.5 \mathrm{~m}, 25 \mathrm{~m}, 27.5 \mathrm{~m}$, and $30 \mathrm{~m}$, whereas their first natural frequencies were 8.79, $6.94,5.62,4.65$, and $3.90 \mathrm{~Hz}$, respectively.

The calculated IMF under different cases is shown in Figure 11. For different bridge spans and road roughness 


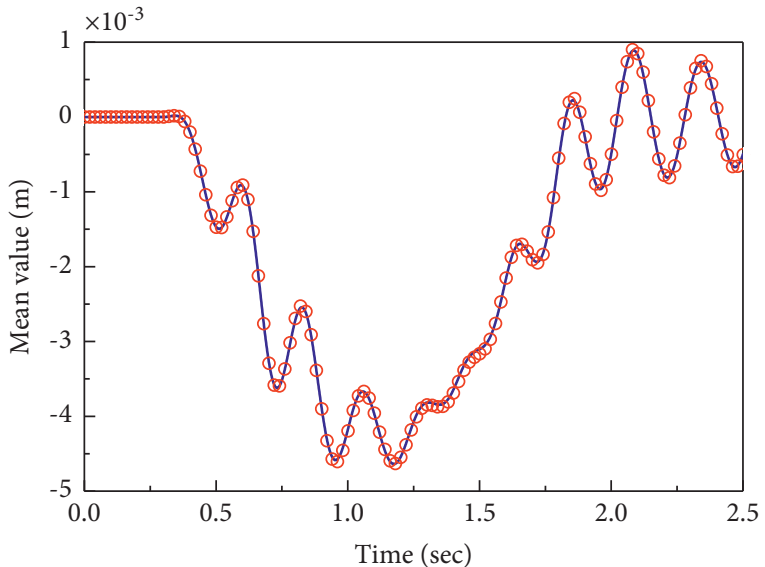

- MCS

- KLE-PEM

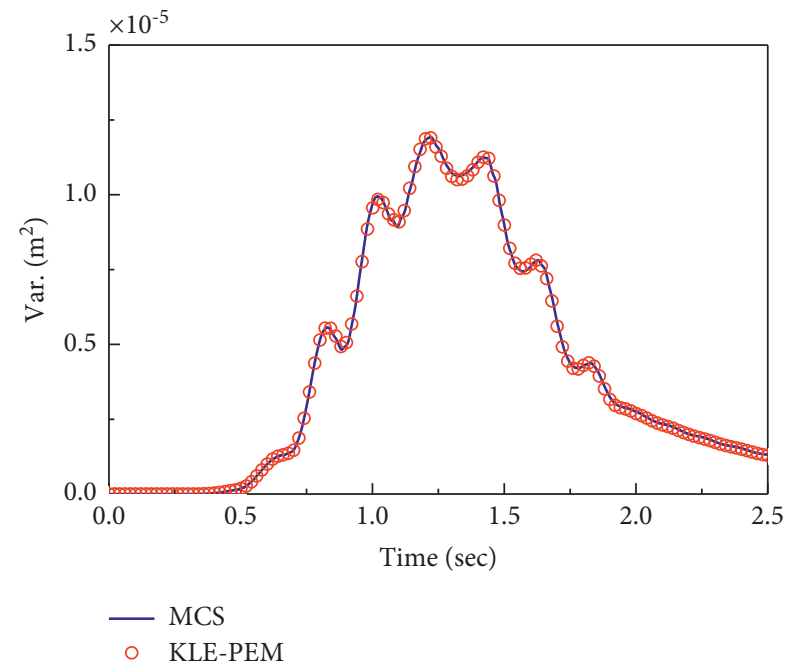

(b)

Figure 6: Comparison of the statistical moment of bridge midspan displacement of the two methods: (a) MEAN; (b) VAR.

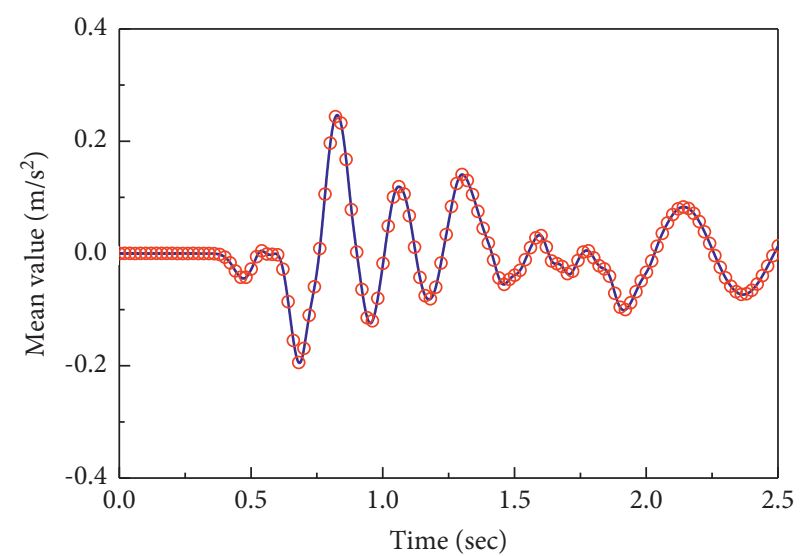

- MCS

○ KLE-PEM

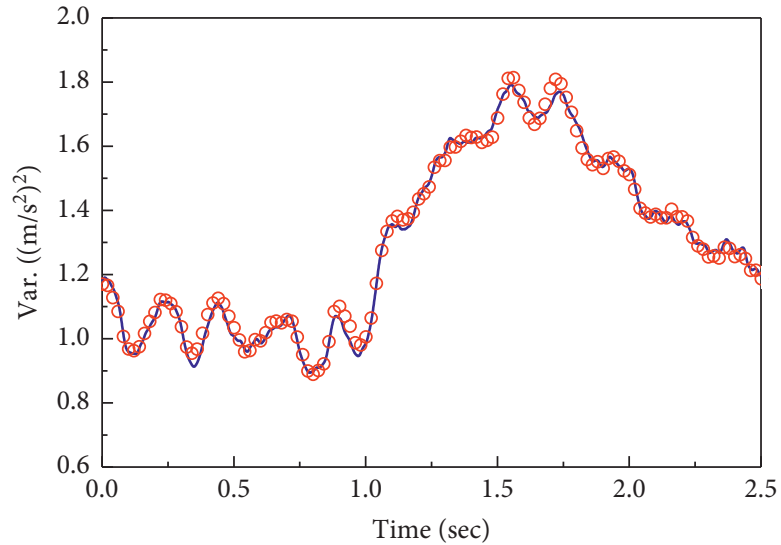

- MCS

- KLE-PEM

(a)

(b)

FIgURE 7: Comparison of the statistical moment of car body vertical acceleration of the two methods: (a) MEAN; (b) VAR.

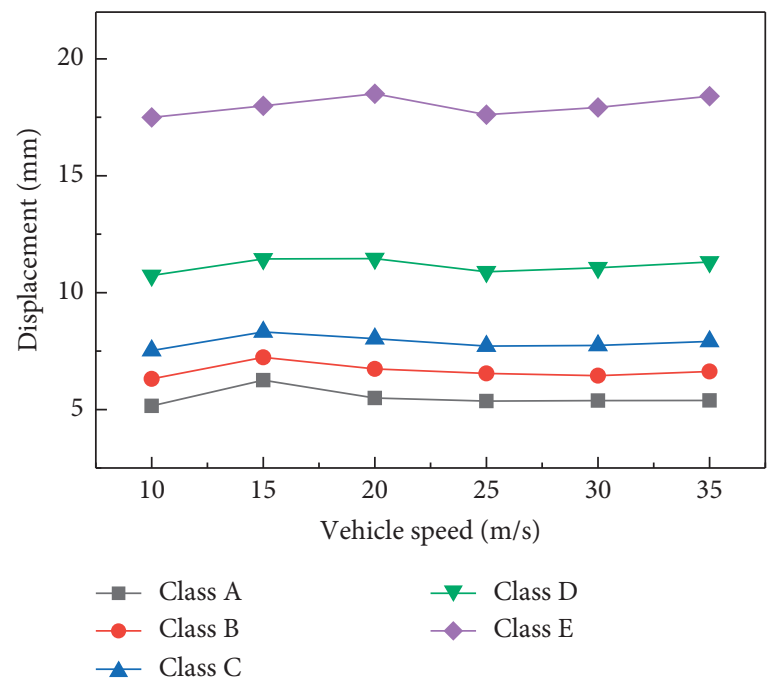

FIgURE 8: Maximum response of bridge displacement via various vehicle and roughness classes. 


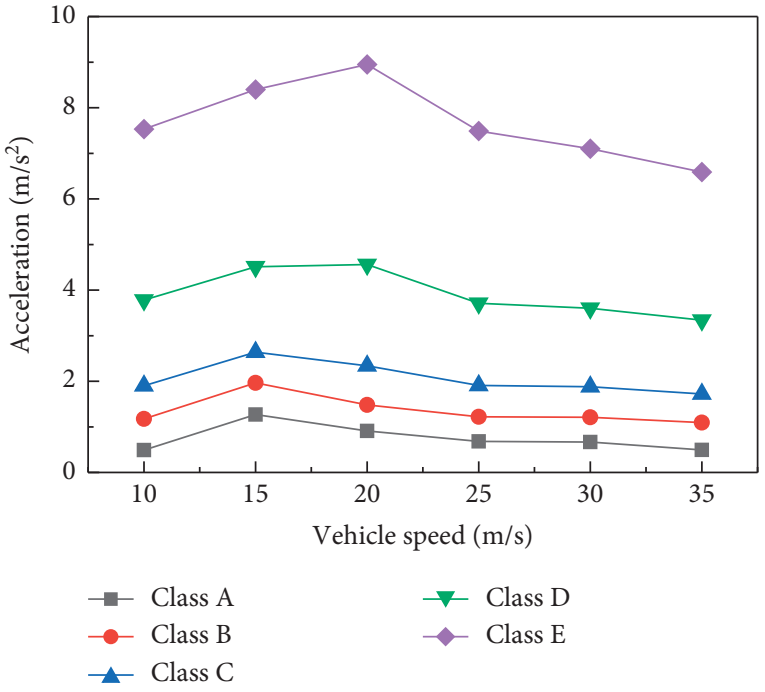

Figure 9: Maximum response of bridge acceleration via various vehicle and roughness classes.

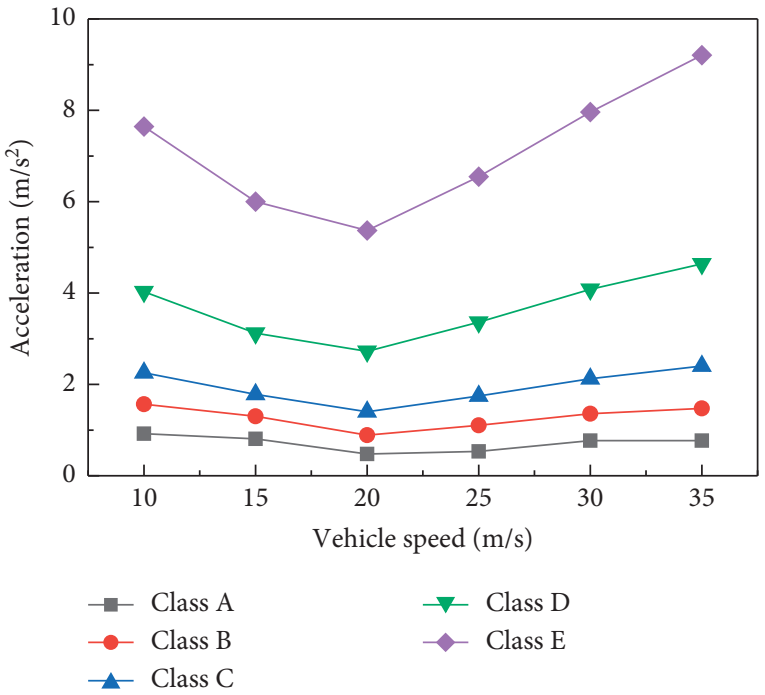

Figure 10: Maximum response of vehicle acceleration via various vehicle and roughness classes.

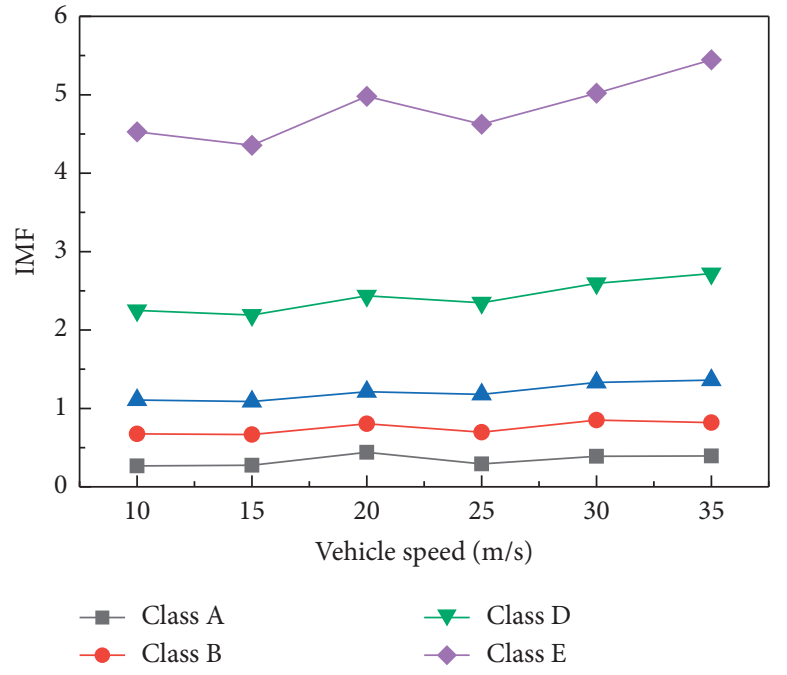

(a)

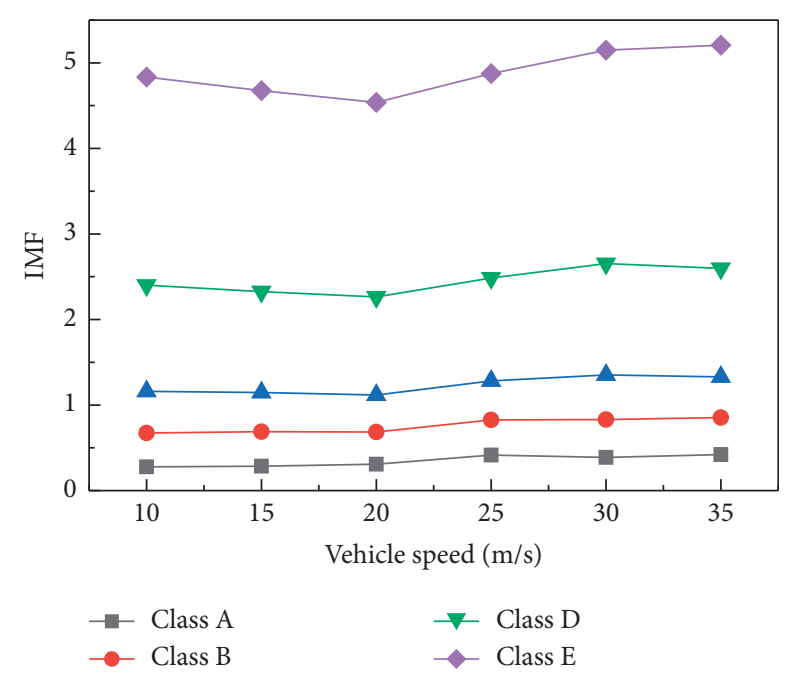

(b)

Figure 11: Continued. 


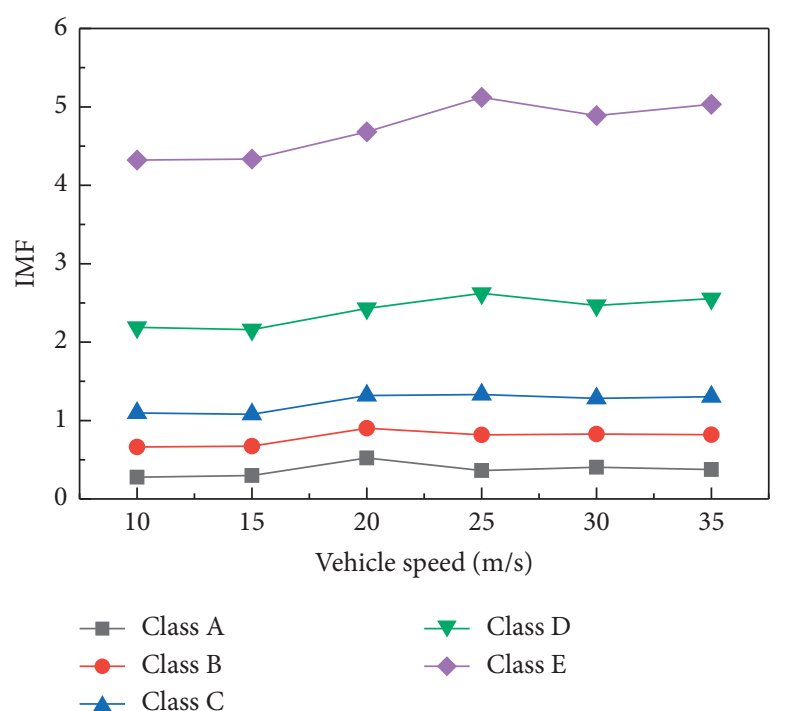

(c)

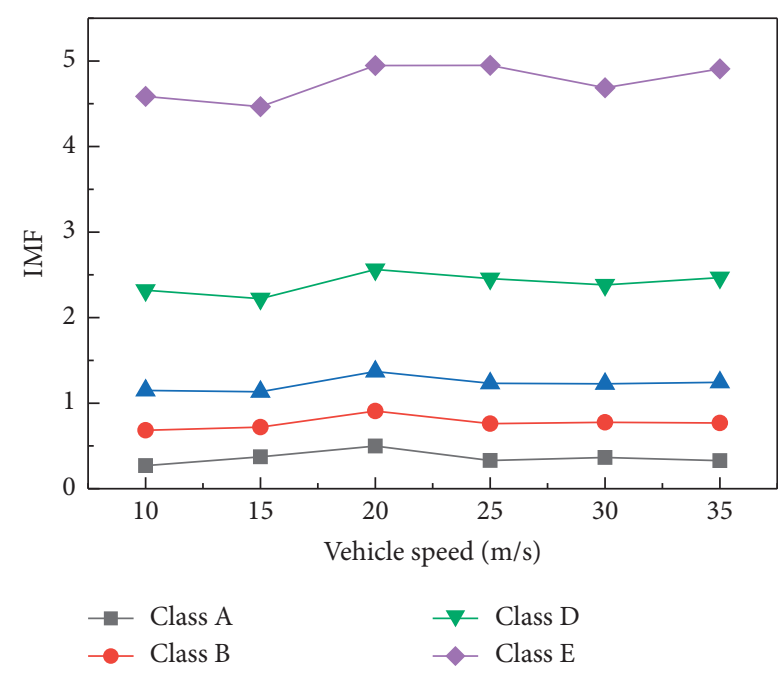

(d)

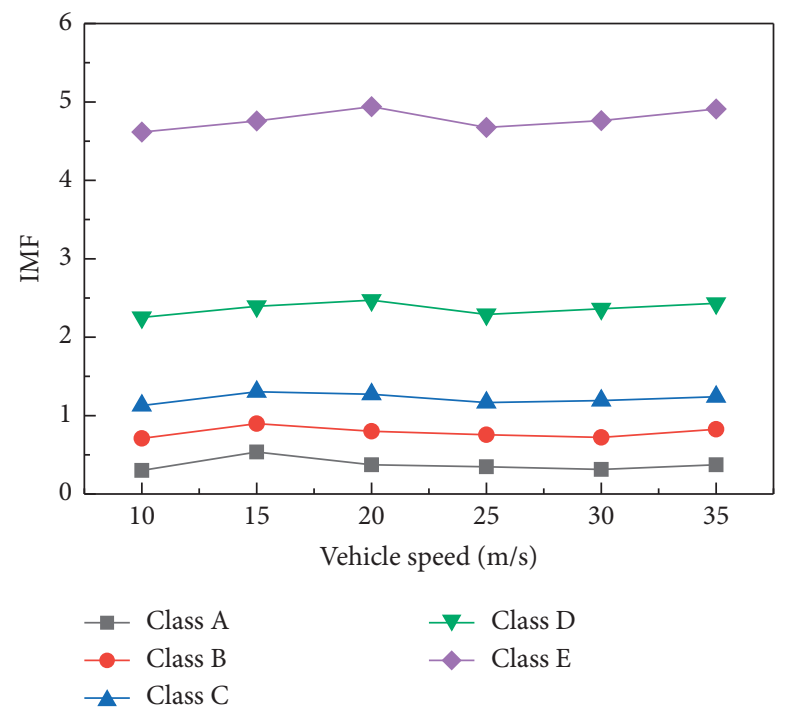

(e)

Figure 11: IMF under different cases: (a) $20 \mathrm{~m}$ span; (b) $22.5 \mathrm{~m}$ span; (c) $25 \mathrm{~m}$ span; (d) $27.5 \mathrm{~m}$ span; (e) $30 \mathrm{~m}$ span.

grades, the IMF of the bridge generally increased with the increase of vehicle speed. For different bridge spans, the change of IMF was not particularly obvious, while the influence of road roughness on IMF was strong. For class A and class $B$ roughness, the IMF was smaller than 1 , while the IMF value was larger than 5 for class $E$. With the growth of the bridge operation time, it was found that the pavement roughness gradually increases, which is why the IMF of the bridge will also gradually increase over time, thus jeopardizing the bridge's structural safety. Therefore, when maintaining the bridge, measurement and maintenance of the pavement roughness should be given due attention.

\section{Conclusions}

To comprehensively analyze the influence of road roughness on the response of the BVI system, a random BVI model considering the randomness of road roughness was established. The vehicle model was simulated by a mass-springdamping system, and the bridge was simulated by the FEM theory. The time-varying system equations of the two were obtained by the energy variational principle. Karhunen-Loeve expansion was used to express the road roughness, and the response's statistical moment was calculated by the PEM. Subsequently, the response of the BVI system with different speeds, different spans, and different roughness was analyzed. The results are as follows:

(1) The KLE-PEM method can obtain the random response of the BVI quickly and precisely, and the computational efficiency is two orders of magnitude higher than that of MCS method.

(2) The sensitivity of bridge displacement and acceleration response to the road roughness is higher than 
the vehicle speed. In addition, the coarser the road surface, the more obvious the influence of vehicle speed change on the bridge response.

(3) Vehicle speed has a great influence on vehicle acceleration, but it does not increase linearly with the increase of vehicle speed; on the other hand, road roughness has a great influence on vehicle acceleration.

(4) Compared with different speeds, different bridge spans, and different pavement roughness, road roughness has a strong influence on the IMF of the bridge, which should elicit sufficient attention when carrying out bridge engineering maintenance.

\section{Data Availability}

The data used to support the findings of this study are included within the article.

\section{Conflicts of Interest}

The authors declare that they have no conflicts of interest.

\section{Acknowledgments}

This work was supported by a grant from the National Natural Science Foundation of China (Grant no. 12072375).

\section{References}

[1] Z. Lai, L. Jiang, and W. Zhou, "An analytical study on dynamic response of multiple simply supported beam system subjected to moving loads," Shock and Vibration, vol. 2018, Article ID 2149251, 14 pages, 2018.

[2] Y. Feng, L. Jiang, and W. Zhou, "Dynamic response of a threebeam system with intermediate elastic connections under a moving load/mass-spring," Earthquake Engineering and Engineering Vibration, vol. 19, pp. 377-395, 2020.

[3] J. Oliva, J. M. Goicolea, P. Antolín, and M. Á. Astiz, "Relevance of a complete road surface description in vehicle-bridge interaction dynamics," Engineering Structures, vol. 56, pp. 466-476, 2013.

[4] Q. Zou, L. Deng, T. Guo, and X. Yin, "Comparative study of different numerical models for vehicle-bridge interaction analysis," International Journal of Structural Stability and Dynamics, vol. 16, Article ID 1550057, 2016.

[5] Q. Zou, L. Deng, and C. Jiang, "Predicting the bounds of vehicle-induced bridge responses using the interval analysis method," Journal of Bridge Engineering, vol. 21, Article ID 04016046, 2016.

[6] H. Wang, T. Nagayama, B. Zhao, and D. Su, "Identification of moving vehicle parameters using bridge responses and estimated bridge pavement roughness," Engineering Structures, vol. 153, pp. 57-70, 2017.

[7] H. Zhong and M. Yang, "Dynamic effect of foundation settlement on bridge-vehicle interaction," Engineering Structures, vol. 135, pp. 149-160, 2017.

[8] X. Liu, L. Jiang, P. Xiang, Z. Lai, Y. Feng, and S. Cao, “Dynamic response limit of high-speed railway bridge under earthquake considering running safety performance of train," Journal of Central South University, vol. 28, no. 3, pp. 968-980, 2021.
[9] L. Xu, Z. Li, Y. Zhao, Z. Yu, and K. Wang, "Modelling of vehicle-track related dynamics: a development of multi-finiteelement coupling method and multi-time-step solution method," Vehicle System Dynamics, pp. 1-28, 2020.

[10] L. Xu and X. Liu, "Matrix coupled model for the vehicle-track interaction analysis featured to the railway crossing," $M e$ chanical Systems and Signal Processing, vol. 152, Article ID 107485, 2021.

[11] L. Xin, X. Li, J. Zhang, Y. Zhu, and L. Xiao, "Resonance analysis of train-track-bridge interaction systems with correlated uncertainties," International Journal of Structural Stability and Dynamics, vol. 20, Article ID 2050008, 2020.

[12] L. Xin, X. Li, Y. Zhu, and M. Liu, "Uncertainty and sensitivity analysis for train-ballasted track-bridge system," Vehicle System Dynamics, vol. 58, no. 3, pp. 453-471, 2020.

[13] P. Xiang, W. Huang, L. Jiang, D. Lu, X. Liu, and Q. Zhang, "Investigations on the influence of prestressed concrete creep on train-track-bridge system," Construction and Building Materials, vol. 293, Article ID 123504, 2021.

[14] S. Q. Wu and S. S. Law, "Dynamic analysis of bridge-vehicle system with uncertainties based on the finite element model," Probabilistic Engineering Mechanics, vol. 25, no. 4, pp. 425432, 2010.

[15] S. Q. Wu and S. S. Law, "Dynamic analysis of bridge with nonGaussian uncertainties under a moving vehicle," Probabilistic Engineering Mechanics, vol. 26, no. 2, pp. 281-293, 2011.

[16] S. Q. Wu and S. S. Law, "Vehicle axle load identification on bridge deck with irregular road surface profile," Engineering Structures, vol. 33, no. 2, pp. 591-601, 2011.

[17] S. Q. Wu and S. S. Law, "Evaluating the response statistics of an uncertain bridge-vehicle system," Mechanical Systems and Signal Processing, vol. 27, pp. 576-589, 2012.

[18] G. Chen and D. Yang, "Direct probability integral method for stochastic response analysis of static and dynamic structural systems," Computer Methods in Applied Mechanics and Engineering, vol. 357, Article ID 112612, 2019.

[19] G. Chen, Z. Meng, and D. Yang, "Exact nonstationary responses of rectangular thin plate on Pasternak foundation excited by stochastic moving loads," Journal of Sound and Vibration, vol. 412, pp. 166-183, 2018.

[20] X. Li, G. Chen, H. Cui, and D. Yang, "Direct probability integral method for static and dynamic reliability analysis of structures with complicated performance functions," Computer Methods in Applied Mechanics and Engineering, vol. 374, Article ID 113583, 2021.

[21] L. Xu, W. Zhai, and J. Gao, "A probabilistic model for track random irregularities in vehicle/track coupled dynamics," Applied Mathematical Modelling, vol. 51, pp. 145-158, 2017.

[22] L. Xu, W. Zhai, and Z. Li, “A coupled model for train-trackbridge stochastic analysis with consideration of spatial variation and temporal evolution," Applied Mathematical Modelling, vol. 63, pp. 709-731, 2018.

[23] L. Xu and W. Zhai, "A novel model for determining the amplitude-wavelength limits of track irregularities accompanied by a reliability assessment in railway vehicle-track dynamics," Mechanical Systems and Signal Processing, vol. 86, pp. 260-277, 2017.

[24] L. Xu and W. Zhai, "Stochastic analysis model for vehicletrack coupled systems subject to earthquakes and track random irregularities," Journal of Sound and Vibration, vol. 407, pp. 209-225, 2017.

[25] L. Xu, W. Zhai, J. Gao, M. Meacci, and X. Chen, "On effects of track random irregularities on random vibrations of 
vehicle-track interactions," Probabilistic Engineering $\mathrm{Me}$ chanics, vol. 50, pp. 25-35, 2017.

[26] X. Liu, P. Xiang, L. Jiang, Z. Lai, T. Zhou, and Y. Chen, "Stochastic analysis of train-bridge system using the $\mathrm{kl}$ expansion and the point estimate method," International Journal of Structural Stability and Dynamics, vol. 20, no. 2, Article ID 2050025, 2020.

[27] X. Liu, L. Jiang, Z. Lai, P. Xiang, and Y. Chen, "Sensitivity and dynamic analysis of train-bridge coupled system with multiple random factors," Engineering Structures, vol. 221, Article ID 111083, 2020.

[28] X. Liu, L. Jiang, P. Xiang et al., "Probability analysis of trainbridge coupled system considering track irregularities and parameter uncertainty," Mechanics Based Design of Structures and Machines, pp. 1-18, 2021.

[29] L. Jiang, X. Liu, P. Xiang, and W. Zhou, “Train-bridge system dynamics analysis with uncertain parameters based on new point estimate method," Engineering Structures, vol. 199, Article ID 109454, 2019.

[30] X. Liu, L. Jiang, P. Xiang, W. Zhou, Z. Lai, and Y. Feng, "Stochastic finite element method based on point estimate and Karhunen-Loéve expansion," Archive of Applied Mechanics, vol. 91, 2021.

[31] C. Cui, Y. L. Xu, Q. H. Zhang, and F. Y. Wang, "Vehicleinduced dynamic stress analysis of orthotropic steel decks of cable-stayed bridges," Structure and Infrastructure Engineering, vol. 16, no. 8, pp. 1067-1081, 2020.

[32] P. Lou and Q. Zeng, "Formulation of equations of motion of finite element form for vehicle-track-bridge interaction system with two types of vehicle model," International Journal for Numerical Methods in Engineering, vol. 62, no. 3, pp. 435-474, 2005.

[33] Y. G. Zhao and T. Ono, "New point estimates for probability moments," Journal of Engineering Mechanics, vol. 126, no. 4, pp. 433-436, 2000.

[34] L. Jiang, X. Liu, T. Zhou et al., “Application of KLE-PEM for random dynamic analysis of nonlinear train-track-bridge system," Shock and Vibration, vol. 2020, Article ID 8886737, 10 pages, 2020.

[35] GB/T 7031-2005, "Mechanical vibration-road surface profilesreporting of measured data," 2005, in Chinese.

[36] Y. B. Yang and J. D. Yau, "Vehicle-bridge interaction element for dynamic analysis," Journal of Structural Engineering, vol. 123, no. 11, pp. 1512-1518, 1997. 